\title{
Direct Observation of SRO effect of Ti-6Al Alloy Using Energy-filtered TEM and Scanning Nanobeam Electron Diffraction
}

\author{
Ruopeng Zhang ${ }^{1,2}$, Rachel Traylor ${ }^{1,2}$, Thomas Pekin ${ }^{1,2}$, Burak Ozdol' ${ }^{2}$, Colin Ophus ${ }^{2}$ and Andrew M. \\ Minor $^{1,2}$ \\ 1. Department of Materials Science and Engineering, University of California, Berkeley, CA, USA \\ 2. National Center for Electron Microscopy, Molecular Foundry, Lawrence Berkeley National \\ Laboratory, Berkeley, CA, USA
}

Titanium alloys are widely used as structural materials due to their high specific strength and excellent corrosion resistance, with $\mathrm{Al}$ as one of the major solid solution strengtheners of $\alpha$-Titanium alloys [1]. The existence of chemical short-range order (SRO) of Ti-Al has been proposed to explain the solidsolution strengthening and low strain hardening [2-3]. Despite extensive research efforts, direct evidence of SRO has only been revealed experimentally by diffuse neutron diffraction measurements [4]. Direct TEM observation of SRO effects would provide insights into understanding the correlations between SRO and deformation behavior.

It has been proposed that "diffuse" anti-phase boundaries are created as dislocations slip through ordered clusters, thereby leading to the localization of plasticity and low strain hardening [5]. Neeraj et al. [6] reported a residual strain in the localized slip bands resulting from this effect, but the direct evidence of SRO disturbance had not been shown. One factor that has limited the utility of TEM on Ti-Al SRO is the low intensity of diffuse superlattice diffraction corresponding to the clusters of short range order. Here, we use two methods in an attempt to directly image the destruction of SRO by dislocation motion in Ti-Al alloys. First, we use an in-column energy filter to eliminate contrast from inelastic scattering. Figure 1 shows the diffuse scattering of $\mathrm{Ti}_{3} \mathrm{Al}$ superlattice indicating the existence of SRO. Dark-field images taken with SRO diffuse scattering peaks (eg. Figure 2) show an evenly distributed darkened contrast. We speculate that the lack of SRO and the residual strain is the origin of this contrast. Second, we use ex situ scanning nanobeam electron diffraction (NBED) to assess the residual strain left in planar slip band and any destruction of the SRO by plasticity. Figure 3 demonstrates an example of an evenly distributed residual $\sim 2 \%$ strain in the basal plane but almost no strain in the prismatic plane. These observations will be discussed in light of the origins of localized plasticity in Ti-Al alloys [8].

\section{References:}

[1] Partridge, P. G., Metallurgical reviews 12.1 (1967), p. 169-194.

[2] M.J. Blackburn and J.C. Williams, Trans. ASM 62 (1969), p. 398-409.

[3] Conrad, H., Scripta Metallurgica 7.5 (1973), p. 509-512.

[4] Fitzner, Arnas, et al., Acta Materialia 103 (2016), p. 341-351.

[5] Van de Walle, A., and M. Asta, Metallurgical and Materials Transactions A 33.3 (2002), p. 735-741.

[6] Neeraj, T., and M. J. Mills, Philosophical Magazine A 82.4 (2002), p. 779-802.

[7] Pekin, Thomas C., et al., Scripta Materialia 146 (2018), p. 87-90.

[8] We gratefully acknowledge funding from the US Office of Naval Research under Grant No.

N00014-12-1-0413. Work at the Molecular Foundry was supported by the Office of Science, Office of Basic Energy Sciences, of the U.S. Department of Energy under Contract No. DE-AC02-05CH11231. 


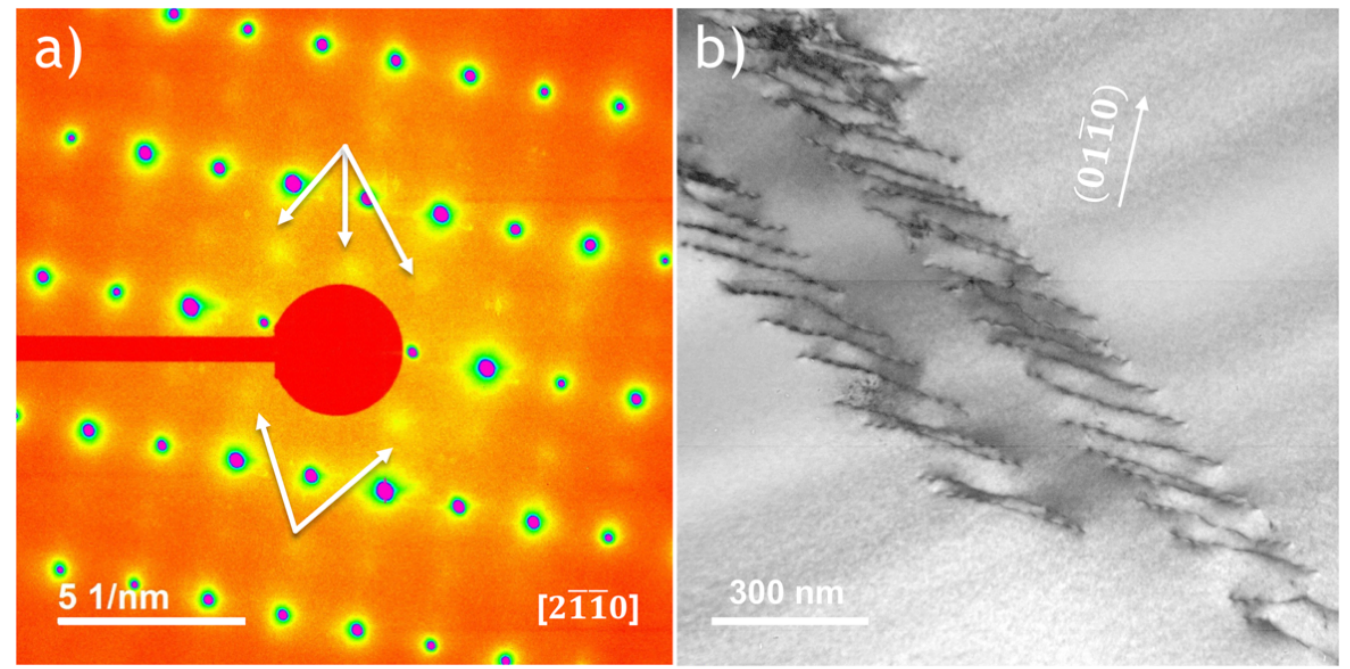

Figure 1. a) Diffraction pattern of aged Ti-6Al alloy showing the diffuse scattering indicative of SRO. b) Bright-field TEM image of localized planar slip bands of the same sample.
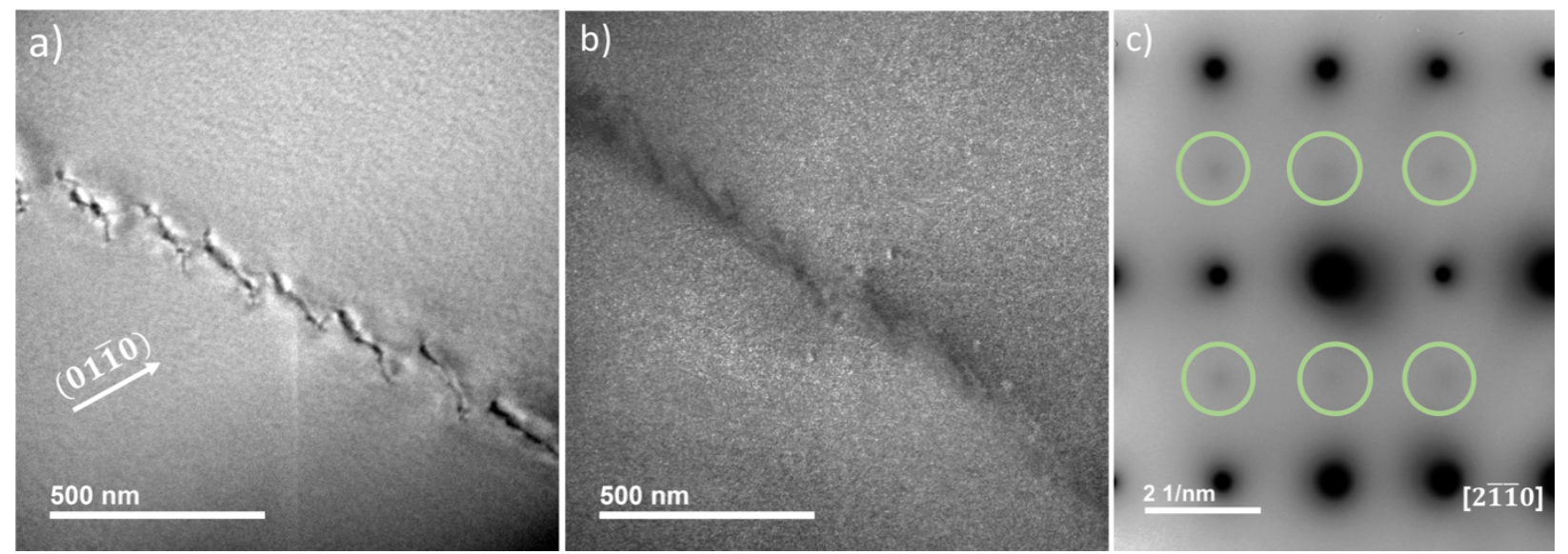

Figure 2. a) Bright-filed TEM image of planar slip. b) Averaged dark-field image using aperture positions indicated in c). c) Diffraction pattern with green circles indicating the aperture positions used for $b)$.
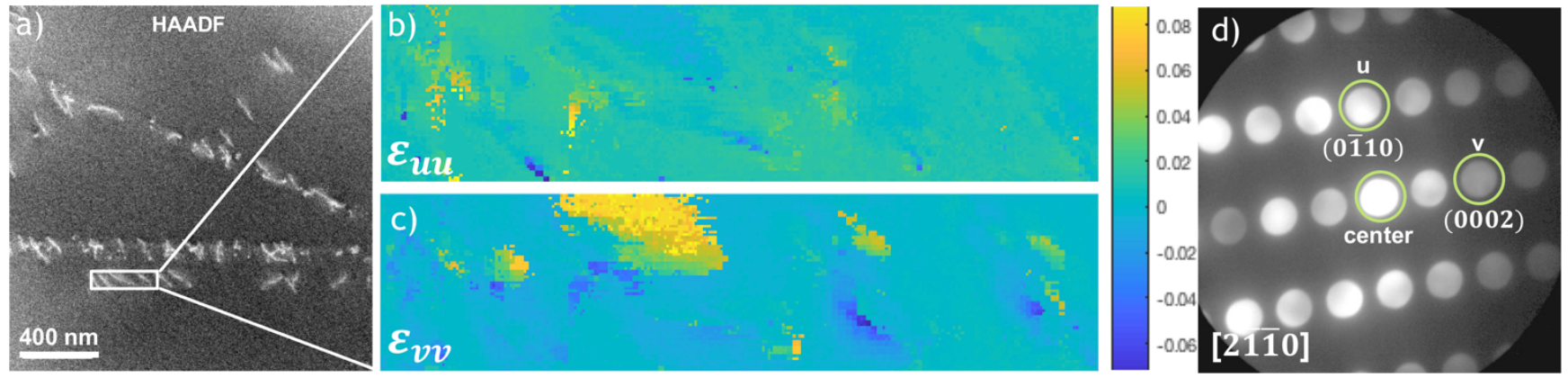

Figure 3. a) High angle annular dark field image showing the position of the planar slip band. b-c) Strain maps in the slip band in orthogonal directions. d) Averaged diffraction pattern showing the direction of $\mathrm{u}, \mathrm{v}$ and their corresponding indices. 